responsibility on public bodies such as Royal Colleges to address discrimination in training and assessment. Understanding DA in undergraduate medical education can help understand DA in the postgraduate setting. Consequently, this systematic review aims to detect the processes that enable and impede DA in UK undergraduate medical education.

Method. Seven online databases including PubMed, Scopus, PyschInfo, and ERIC were searched. A formal grey literature search was also conducted. Inclusion criteria comprised studies dated from January 1995 to present and included UK undergraduate medical students. We present the preliminary findings from 13 papers, analysed to create a conceptual framework for a further mixed methods analysis. The studies were critically appraised for methodological quality.

Result. Five key themes emerged from the preliminary analysis of 13 papers. BAME students experienced:

Being 'divergent': Not feeling part of the current organisational learning milieu

Lack of social capital: Difficulty in being absorbed into existing 'networks' of relationships in a manner that is 'approachable' and not 'intimidating'

Continuum of discrimination: 'Indirect' impact of subtle communication processes in the learning environment undermining individual 'belief' in own performance

Institutional discriminatory factors: Culture, rules, norms, and behavioural routines of educators that lead to differential outcomes for learners

Lack of external support: Relative lack of interventions tackling DA.

Conclusion. The key finding of this review is that British BAME undergraduate medical students experience discriminatory behaviours early in medical schools that impact on personal, educational, and professional outcomes. These factors may need to be borne in mind by postgraduate training organisations such as the Royal College of Psychiatrists as they commence the challenging task of addressing DA.

\section{Improving the safety of rapid tranquilisation in older} people

Richard Harris ${ }^{\star}$, Rollo Sheldon, Jane McNulty and Scott Cherry Sussex Partnership NHS Trust

${ }^{*}$ Corresponding author.

\section{doi: 10.1192/bjo.2021.129}

Aims. To identify intramuscular rapid tranquilisation (IMRT) events in all $>65$ years inpatients in Sussex Partnership NHS Foundation Trust (SPFT) and to establish whether accompanying documentation meets SPFT guidelines. This is a re-audit, initial data were collected in 2016. Multimodal intervention has been implemented since initial data collection. In psychiatric inpatients IMRT should be administered as a last resort to calm acutely disturbed patients after verbal de-escalation and an offer of oral medication has failed. IMRT can cause physical health complications and impact therapeutic relationships. Quality improvements made since initial data collection were: an IMRT treatment algorithm for $>65 \mathrm{~s}$, a teaching package for staff, IMRT prescription area on medicine cards and post IMRT physical monitoring forms - in line with updates to trust IMRT policy.

Method. Retrospective case note audit cycle of 119 patients. Electronic and paper records were reviewed for inpatients $>65$ years on 1/9/2019. Records were examined for instances of IMRT - the following features were noted: diagnosis; verbal de-escalation; oral medication offered prior to IMRT; IMRT prescription location; and post-IMRT monitoring. Descriptive statistics were performed. This audit was approved by the trust audit committee.

Result. There were 34 RT events in 17 patients, reduced from $83 \mathrm{RT}$ events in 20 patients in 2016. De-escalation was attempted in $62 \%$ versus $34 \%$ in 2016 , oral medication offered first in $71 \%$ versus $59 \%$ in 2016. Physical monitoring was fully completed in $50 \%$ of instances in 2019, an improvement from $23 \%$ in 2016.

Conclusion. Education, a new treatment algorithm, medicine card changes, and IMRT physical monitoring forms have improved adherence to trust standards. There was a $49 \%$ reduction in IMRT events in 2019 versus 2016. De-escalation is being performed more frequently, and oral sedation offered in more cases. The physical monitoring of patients has improved.

\section{The psychiatry virtual-on-call experience: Can it} improve confidence of foundation and GP trainees with out-of-hours work in psychiatry?

Charlotte Harrison $^{1 \star}$, Helen Blamey ${ }^{1}$, Alistair Roddick ${ }^{1}$, Kate Saunders ${ }^{2}$ and Tina Malhotra ${ }^{3}$

${ }^{1}$ OUCAGS (Oxford University Clinical Academic Graduate School), University of Oxford, Oxford Health NHS Foundation Trust;

${ }^{2}$ OUCAGS (Oxford University Clinical Academic Graduate School), University of Oxford, Department of Psychiatry, University of Oxford, Warneford Hospital and ${ }^{3}$ Oxford Health NHS Foundation Trust

${ }^{\star}$ Corresponding author.

doi: 10.1192/bjo.2021.130

Aims. Out-of-hours ('on-call') work can be perceived by junior doctors to be a daunting experience, associated with feeling unprepared and less supported. Simulated on-call programmes have been used to great effect in medicine and surgery to improve junior doctors' skills in task prioritisation, interpersonal communication and confidence on-call. However, few psychiatry-specific programmes exist.

We aimed to: i) Develop a psychiatry specific virtual-on-call programme, ii) Investigate if the virtual-on-call programme improved confidence amongst junior trainees in key areas of psychiatry practice.

Method. The Psychiatry Virtual-On-Call programme commenced in December 2020. It involves attending an introductory on-call lecture, followed later in the rotation by a 2-hour simulated on-call shift. All trainees are expected to attend during their attachment and the simulated shifts are ongoing. During the shift, trainees are 'bleeped' with different psychiatry specific tasks. They work through the tasks, using local intranet policies and telephone advice from the on-call psychiatry registrar. Due to COVID-19 the sessions were delivered virtually. Participants completed a questionnaire evaluating confidence in ten domains, rated on a Likert scale from 0-10. Questionnaires were completed at four time-points during the programme; pre- and postintroductory lecture and pre- and post-simulated shift. Scores were compared using Mann-Whitney U tests. Significance was defined as $\mathrm{P}<0.05$ with Bonferroni correction applied for multiple testing.

Result. Twenty-nine trainees attended the introductory lecture, 25 and 21 trainees completed the pre- and post-lecture questionnaire respectively. A non-significant improvement in confidence was reported in three domains: seclusions reviews, prescribing, detention under the mental health act. 
At the time of writing, ten trainees had attended the on-call shift. All participants completed a pre- and post-session questionnaire. The on-call shift was a useful learning experience (median score 9), and significantly increased perceived preparedness for on-call work from $3 / 10$ to $7 / 10(p<0.001)$. Confidence was significantly improved in seven domains, most markedly in seclusion reviews, prescribing and mental health act tasks.

Conclusion. The psychiatry virtual-on-call programme fills a niche in the training curriculum and is perceived by trainees to be a useful learning experience. The introductory lecture improved confidence in several domains, but not as effectively as the on-call shift. The on-call shift was well received by participants and significantly improved confidence in 7/10 domains. In summary, the virtual-on-call experience improves preparedness for out-of-hours psychiatry work. Follow-up of participants at the end of their psychiatry rotation will ascertain if they felt the programme to be useful during out-of-hours work.

An analysis of lithium requesting across three hospital trusts in the UK: many people are managed with lithium levels below the current nice guidance lower limit

Adrian Heald ${ }^{1 \star}$, Ceri Parfitt ${ }^{2}$, Chris Duff ${ }^{3}$, Jonathan Scargill ${ }^{4}$, Lewis Green ${ }^{5}$ and Anthony Fryer ${ }^{3}$

${ }^{1}$ University of Manchester; ${ }^{2}$ Royal Stoke University Hospital; ${ }^{3}$ Royal Stoke Hospital; ${ }^{4}$ North Manchester General Hospital and ${ }^{5}$ St Helens and Knowsley Hospital

${ }^{*}$ Corresponding author.

doi: 10.1192/bjo.2021.131

Aims. This study examined lithium results and requesting patterns over a 6-year period, and compared these to guidance.

Background. Bipolar disorder is the 4 th most common mental health condition, affecting $\sim 1 \%$ of UK adults. Lithium is an effective treatment for prevention of relapse and hospital admission, and is recommended by NICE as a first-line treatment.

We have previously shown in other areas that laboratory testing patterns are highly variable with sub-optimal conformity to guidance.

Method. Lithium requests received by Clinical Biochemistry Departments at the University Hospitals of North Midlands, Salford Royal Foundation Trust and Pennine Acute Hospitals from 2012-2018 were extracted from Laboratory Information and Management Systems (46,555 requests; 3,371 individuals). We categorised by request source, lithium concentration and re-test intervals.

Result. Many lithium results were outside the NICE therapeutic window $(0.6-0.99 \mathrm{mmol} / \mathrm{L}) ; 49.3 \%$ were below the window and $6.1 \%$ were above the window (median $[\mathrm{Li}]: 0.61 \mathrm{mmol} / \mathrm{L}$ ). A small percentage were found at the extremes $(3.2 \%$ at $<0.1 \mathrm{mmol} / \mathrm{L}$, $1.0 \%$ at $>1.4 \mathrm{mmol} / \mathrm{L})$. Findings were comparable across all sites.

For requesting interval, there was a distinct peak at 12 weeks, consistent with guidance for those stabilised on lithium therapy. There was no peak evident at 6 months, as recommended for those $<65$ years old on unchanging therapy. There was a peak at 0-7 days, reflecting those requiring closer monitoring (e.g. treatment initiation or results suggesting toxicity).

However, $77.6 \%$ of tests were requested outside expected testing frequencies.

Conclusion. We showed: (a) lithium levels are often maintained at the lower end of the NICE recommended therapeutic range (and the BNF range: $0.4-1.0 \mathrm{mmol} / \mathrm{L}$ ); (b) patterns of lithium results and testing frequency are comparable across three sites with differing models of care; (c) re-test intervals demonstrate a noticeable peak at the recommended 3-monthly interval, but not at 6-monthly intervals; (d) Many tests were repeated outside these expected frequencies (contrary to NICE guidance).

\section{We can check serum lithium levels less often without compromising patient safety: evidence from a multi-centre study}

Adrian Heald ${ }^{1 \star}$, David Holland ${ }^{2}$, Mark Davies ${ }^{3}$, Chris Duff ${ }^{4}$, Ceri Parfitt ${ }^{4}$, Lewis Green ${ }^{5}$, Jonathan Scargill ${ }^{6}$, David Taylor ${ }^{7}$ and Anthony Fryer ${ }^{8}$

${ }^{1}$ University of Manchester; ${ }^{2}$ The Benchmarking Partnership; Mike Stedman, Res Consortium; ${ }^{3}$ Res Consortium; ${ }^{4}$ University Hospitals of North Midlands NHS Trust; ${ }^{5}$ St. Helens \& Knowsley Teaching Hospitals NHS Trust; ${ }^{6}$ Royal Oldham Hospital, Oldham, Pennine Care NHS Foundation Trust; ${ }^{7}$ Institute of Psychiatry, Psychology and Neuroscience, King's College London and ${ }^{8}$ Keele University ${ }^{\star}$ Corresponding author.

doi: 10.1192/bjo.2021.132

Aims. Lithium was first found to have an acute antimanic effect in 1948 with further corroboration in the early 1950s. It took some time for lithium to become the standard treatment for relapse prevention in bipolar affective disorder. In this study, our aims were to examine the factors associated wtih the likelihood of maintaining lithium levels within the recommended therapeutic range and to look at the stability of lithium levels between blood tests. We examined this relation using clinical laboratory serum lithium test requesting data collected from three large UK centres, where the approach to managing patients with bipolar disorder and ordering lithium testing varied.

Method. 46,555 lithium rest requests in 3,371 individuals over 7 years were included from three UK centres. Using lithium results in four categories $(<0.4 \mathrm{mmol} / \mathrm{L} ; \quad 0.40-0.79 \mathrm{mmol} / \mathrm{L}$; 0.80-0.99 $\mathrm{mmol} / \mathrm{L} ; \geq 1.0 \mathrm{mmol} / \mathrm{L}$ ), we determined the proportion of instances where, on subsequent testing, lithium results remained in the same category or switched category. We then examined the association between testing interval and proportion remaining within target, and the effect of age, duration of lithium therapy and testing history.

Result. For tests within the recommended range (0.40-0.99 $\mathrm{mmol} / \mathrm{L}$ categories), $84.5 \%$ of subsequent tests remained within this range. Overall 3-monthly testing was associated with $90 \%$ of lithium results remaining within range compared with $85 \%$ at 6-monthly intervals. At all test intervals, lithium test result history in the previous 12-months was associated with the proportion of next test results on target (BNF/NICE criteria), with 90\% remaining within range target after 6-months if all tests in the previous 12-months were on target. Age/duration of lithium therapy had no significant effect on lithium level stability. Levels within the $0.80-0.99 \mathrm{mmol} / \mathrm{L}$ category were linked to a higher probability of moving to the $\geq 1.0 \mathrm{mmol} / \mathrm{L}$ category (10\%) than those in the $0.40-0.79$ mmolL group (2\%), irrespective of testing frequency. Thus prior history in relation to stability of lithium level in the previous 12 months is a predictor of future stability of lithium level.

Conclusion. We propose that, for those who achieve 12-months of lithium tests within the $0.40-0.79 \mathrm{mmol} / \mathrm{L}$ range, it would be reasonable to increase the interval between tests to 6 months, irrespective of age, freeing up resource to focus on those less concordant with their lithium monitoring. Where lithium level is 